\title{
Taxonomía de errores y dificultades en la construcción e interpretación de tablas de frecuencia
}

\author{
Taxonomy of errors and difficulties in the construction and interpretation of frequency \\ tables \\ Ingrith Álvarez Alfonso ${ }^{1}$ \\ Yuly Guerrero Gutiérrez ${ }^{2}$ \\ Yessica Torres López ${ }^{3}$
}

\section{Resumen}

Se describe un proceso de investigación cualitativa llevada a cabo con estudiantes de educación básica y media en Colombia. Se tuvo como objetivo corroborar un conjunto de dificultades y errores que se preveían a partir de la práctica pedagógica para los cuales no se hallaba sustento teórico particular. Se estableció la necesidad de centrar la atención en los elementos estructurales de las tablas de frecuencias y su relación con los errores reportados para gráficos estadísticos con el fin de encontrar una explicación teórica de los errores asociados a tablas. Los datos fueron recolectados de un total de 100 estudiantes, mediante el uso de instrumentos diseñados con el propósito de obtener evidencia que permitiera la formulación de una taxonomía de los errores y las dificultades asociadas en la construcción e interpretación de tablas de frecuencia. La taxonomía representa una contribución teórica que puede ser relevante en el estudio de los procesos pedagógicos relacionados con este objeto de estudio, y el diseño gestión de actividades para superar o mitigar tales errores y sus dificultades.

Palabras clave: Errores; Dificultades; Construcción tablas de frecuencia; Interpretación tablas de frecuencia.

\begin{abstract}
This article describes a qualitative research process carried out with secondary school students in Colombia. The research aimed at further exploring a set of errors and difficulties which were identified through the teaching experience and for which no particular theoretical support was found. It established the need to focus on the structural elements of frequency tables and their relation to errors in the corresponding statistical graphs so that a theoretical explanation for the errors could be found. Data was collected from a total of 100 students by using instruments designed purposefully to obtain evidence that allowed formulating a taxonomy of errors and difficulties associated with the construction and interpretation of frequency tables. The taxonomy represents a theoretical contribution that may be relevant in the study of teaching processes related to this subject matter, and the design of teaching activities to overcome or mitigate such errors and difficulties.
\end{abstract}

Keywords: Errors and Difficulties; Construction \& Interpretation of Frequency Tables.

Submetido em: 04/09/2019 - Aceito em: 12/02/2020 - Publicado em: 14/04/2020

${ }^{1}$ Magister en Docencia de la Matemática de la Universidad Pedagógica Nacional. Profesora Investigadora de la Universidad Pedagógica Nacional, Colombia. Email: ialvarez@pedagogica.edu.co

${ }^{2}$ Magister en Docencia de la Matemática de la Universidad Pedagógica Nacional. Profesora de educación básica, Colombia. Email: yaguerrerog@upn.edu.co

${ }^{3}$ Licenciada en Matemáticas de la Universidad Pedagógica Nacional, Colombia. Email: dma_ytorres438@pedagogica.edu.co 


\section{Introducción}

El desarrollo del pensamiento estadístico cumple un papel fundamental en la formación de ciudadanos estadísticamente alfabetizados o cultos. Este pensamiento busca aportar para que todo individuo esté en capacidad de interpretar y analizar de manera crítica la información presente en diversos medios de comunicación. Información en gráficos o tablas estadísticas, datos estadísticos en textos tipo artículo de prensa, editoriales o blogs. Esta alfabetización también contempla la importancia de un conocimiento necesario y suficiente para que los individuos además de ser consumidores críticos de información, puedan asumir roles de productores de la misma. Esto es, tener la capacidad de recoger y organizar datos estadísticos, tanto en tablas de conteo y tablas de frecuencia y de contingencia, y tal y como lo afirma Tauber (2010), generar gráficos estadísticos asociados a conjuntos de datos. Por ende, los conceptos de variable estadística, valor de la variable, frecuencia y sus diferentes clases, y la construcción e interpretación de tablas estadísticas (tablas de frecuencias) se han ido posicionando al interior del currículo escolar colombiano.

Conforme lo describe el MEN (1998) en lo que denomina 'Pensamiento aleatorio y sistemas de datos', el tratamiento de situaciones de la vida cotidiana o de otras ciencias, debe darse a través de la exploración e investigación, involucrando recolección, organización, representación y análisis de datos a través de tablas, cuya interpretación oriente la toma de decisiones (p.47). Asunto que el Ministerio consolida cuando propone que los estudiantes clasifiquen y organicen datos de acuerdo a sus cualidades y los representen usando tablas y gráficas (pictogramas, gráficas de barras, diagramas de líneas, diagramas circulares); interpreten información exhibida en tablas y gráficas estadísticas; y resuelvan y formulen problemas a partir de un conjunto de datos presentados en tablas, diagramas de barras, diagramas circulares u otros (MEN, 2006). Este panorama de formación ciudadana implica que el docente de estadística de la educación básica y media ${ }^{4}$ aborde en el aula objetos de estudio tales como tablas de frecuencia para datos agrupados y no agrupados (fundamento conceptual para la construcción de gráficos estadísticos), y ponga en escena estrategias que aporten al desarrollo del pensamiento aleatorio, de manera particular a las habilidades de construcción e interpretación de dicho tipo de tablas.

Empero estas orientaciones curriculares nacionales, experiencias de práctica educativa muestran que los docentes se encuentran con escenarios poco positivos frente al aprendizaje de tales objetos. Escenarios que en ocasiones no logran ser explicados por las teorías existentes a pesar de que algunos autores destacan la importancia de reconocer y analizar errores, dificultades y obstáculos que se presentan durante los procesos de enseñanzaaprendizaje. Por ejemplo, Batanero (2001) caracteriza estos elementos como insumos vitales para el docente puesto que sirven de fundamento para (re)orientar el proceso pedagógico.

\footnotetext{
${ }^{4}$ La estructura del sistema educativo colombiano está dada por: la educación inicial (antes de los 4 años), la educación preescolar (de los 4 a los 6 años), la educación básica primaria (cinco grados, de los 6 a los 10 años), la educación básica secundaria (cuatro grados, de los 11 a los 14 años) y la educación media (dos grados, culminando con el título de bachiller, de los 15 a los 16 años), y la educación superior.
} 
Socas (1997) manifiesta, de manera particular, que caracterizar errores, dificultades y obstáculos sirve de orientación para el diseño de actividades que apoyen su superación o eviten el surgimiento de los mismos.

Dada la importancia de contar con referentes relacionados con errores y dificultades en la construcción e interpretación de tablas de frecuencia, se asume el reto de desarrollar una investigación cualitativa descriptiva que permita narrar el proceso llevado a cabo con estudiantes de educación básica y media en Colombia. La investigación tuvo como objetivo explicar y exponer teóricamente dificultades y errores que se perciben en las aulas y de los cuales no se hallaba referentes teóricos específicos. Como parte del proceso investigativo se diseñaron e implementaron dos instrumentos (fragmentos presentados en los apéndices) para recoger información. Los datos sirvieron de evidencia para contrastar las hipótesis provenientes del ejercicio docente y algunas analogías propuestas desde la teoría, describir con detalle los errores y las dificultades asociadas a la construcción e interpretación de tablas de frecuencia y formular la taxonomía (aporte teórico al campo investigativo).

\section{Marco de referencia}

Los referentes de la investigación se orientan a partir de las concepciones respecto a lo que se denomina obstáculo, dificultad y error en el contexto de las estructuras conceptuales que se desarrollan al aprender matemáticas. Dichas concepciones se asocian al campo del pensamiento aleatorio y de manera particular a los errores inherentes a la construcción de gráficos estadísticos. Esto se debe a que tradicionalmente se asumen las representaciones de datos desde los gráficos sin centrar la atención en elementos estructurales y constitutivos de las tablas. Enfocarse, como punto de partida, en la construcción de gráficos conlleva a asumir los niveles de interpretación propuestos por Friel, Curcio y Bright (2001) y las habilidades para su comprensión, cimiento para erigir los instrumentos de recolección de la información.

\section{Dificultades, obstáculos y errores}

Socas (1997) señala que las dificultades se conectan y fortalecen en redes conceptuales complejas, se concretan en la práctica como obstáculos y se manifiestan en forma de errores. En este apartado tales nociones se abordan en dicho orden guardando tal relación. Así, se entienden las dificultades como un conflicto que se genera durante el proceso de aprendizaje de las matemáticas y se asume que estas pueden ser de distinta naturaleza, tal y como se clasifican en el Cuadro 1.

Cuadro 1 - Tipos de dificultades

\begin{tabular}{|c|l|}
\hline \multirow{2}{*}{$\begin{array}{c}\text { Dificultades asociadas a } \\
\text { la complejidad del objeto } \\
\text { matemático }\end{array}$} & $\begin{array}{l}\text { Estas dificultades están aunadas a la estructura conceptual del objeto } \\
\text { matemático estudiado (representaciones gráficas o simbólicas, definiciones, } \\
\text { aplicaciones, etc.). Al nivel de abstracción que se logra del mismo. A la } \\
\text { variedad de significados que la terminología asociada al objeto puede tener para } \\
\text { el estudiante según su cotidianidad, puesto que asumir un nuevo significado } \\
\text { para determinada expresión implica modificación y adaptación cognitiva. }\end{array}$ \\
\hline $\begin{array}{c}\text { Dificultades asociadas a } \\
\text { los procesos de }\end{array}$ & $\begin{array}{l}\text { Esta categoría se asocia a las rupturas (modificación y adaptación) necesarias en } \\
\text { relación con los modos de pensamiento matemático. A la necesidad de alcanzar }\end{array}$ \\
\hline
\end{tabular}


DOI: $10.20396 /$ zet.v28i0.8656553

\begin{tabular}{|c|l|}
\hline pensamiento matemático & $\begin{array}{l}\text { procesos de mayor envergadura que implican la deducción lógica formal y } \\
\text { dominar procedimientos algorítmicos para lograr determinado nivel de } \\
\text { competencias matemáticas. }\end{array}$ \\
\hline $\begin{array}{c}\text { Dificultades asociadas a } \\
\text { los procesos de } \\
\text { enseñanza de las } \\
\text { matemáticas }\end{array}$ & $\begin{array}{l}\text { Tales dificultades están directamente asociadas a las decisiones entorno a las } \\
\text { instituciones escolares, el currículo, los métodos de enseñanza, las decisiones } \\
\text { administrativas, y las perspectivas metodologías que se asumen en el aula. Estos } \\
\text { componentes median e impactan en el aprendizaje de los estudiantes. }\end{array}$ \\
\hline $\begin{array}{c}\text { Dificultades asociadas a } \\
\text { los procesos de }\end{array}$ & $\begin{array}{l}\text { Las dificultades de esta categoría se relacionan directamente con el impacto que } \\
\text { tiene en el proceso pedagógico los niveles de razonamiento alcanzados por los } \\
\text { estudiantes, los estadios de desarrollo intelectual y los procesos de aprendizaje } \\
\text { de cada uno de ellos según sus edades y niveles de formación. }\end{array}$ \\
\hline $\begin{array}{c}\text { los alumnos } \\
\text { Dificultades asociadas a } \\
\text { actitudes afectivas y } \\
\text { emocionales hacia las } \\
\text { matemáticas }\end{array}$ & $\begin{array}{l}\text { Aquí se circunscriben las dificultades que tienen que ver con el disgusto de los } \\
\text { estudiantes hacia las matemáticas, las cuales afectan el proceso de enseñanza- } \\
\text { aprendizaje y pueden ser producto de aspectos tales como actitud negativa de } \\
\text { los profesores en el aula, la ansiedad por terminar una tarea que no es del } \\
\text { agrado, el miedo al fracaso o las actitudes y creencias negativas hacia las } \\
\text { matemáticas, que les fueron transmitidas. }\end{array}$ \\
\hline
\end{tabular}

Fuente: Propia inspirada en Socas (1997)

Estos tipos de dificultades conllevan a la aparición de obstáculos. Desde la perspectiva de Bachelard (1988) estos refieren a estancamientos conceptuales fruto de confusiones que se dan cuando no se logra avanzar en el desarrollo de una determinada tarea haciendo uso del conocimiento que se tiene hasta el momento. El obstáculo se identifica cuando un nuevo conocimiento entra en conflicto con uno previo que puede haberse adquirido de manera errónea o parcialmente correcta, y que fue funcional en determinadas situaciones, pero que al usarlo para afrontar una nueva tarea no logra soportar las exigencias conceptuales de la misma y entra en conflicto con la estructura cognitiva del estudiante. En este ámbito se reconocen los obstáculos de origen psicogenético, didáctico y epistemológico. Los de origen psicogenético se relacionan con la etapa de desarrollo del individuo que desafía la situación de aprendizaje. Los de origen didáctico están vinculados con la metodología usada en el proceso de enseñanza-aprendizaje y son fruto esencialmente del proceso pedagógico que llevan a cabo los docentes. Los obstáculos de origen epistemológico se asocian a la dificultad propia del concepto, la cual podría identificarse a través del desarrollo histórico del mismo.

Siguiendo a Socas (1997), la persistencia de dificultades y de obstáculos en el proceso pedagógico y un esquema cognitivo inadecuado genera el error. Este se entiende como la manifestación externa (escrita -simbólica, algorítmica, gráfica-, verbal, actitudinal, etc.) de los obstáculos. El error es visible a los ojos de los docentes pero en pocas ocasiones lo es para los estudiantes, puesto que para ellos su conocimiento sigue siendo eficiente a pesar de no lograr solucionar satisfactoriamente la tarea.

\section{Errores y dificultades: Gráficos estadísticos}

Rodríguez y Sandoval (2012) plantean que los sistemas de representación de datos, particularmente las tablas de frecuencia, desempeñan un rol significativo tanto en el análisis de datos como en la comunicación de los mismos en distintos ámbitos educativos y sociales. 
DOI: $10.20396 /$ zet.v28i0.8656553

A partir de ello se reconoce que tablas y gráficos estadísticos comparten la misma naturaleza, lo que da lugar al hecho de tomar como soporte inicial de la investigación algunos errores que cometen los estudiantes al momento de construir gráficos. Entre ellos, según Batanero y Godino (2002, p. 727) están:

- Elección incorrecta del tipo de gráfico en relación con la variable estadística que se está estudiando y la información que se quiere presentar de la misma y del conjunto de datos.

- La elección de las escalas de representación es poco adecuada en comparación con la cantidad de datos que se presentan.

- Omisión de las escalas de representación en ambos o alguno de los ejes.

- No especificar el origen de coordenadas ya sea para las frecuencias o para los valores que toma la variable estadística.

- Ausencia de suficientes divisiones en las escalas de los ejes, que permitan dar claridad a la información que se representa.

- Mezclar en un mismo gráfico datos que no son comparables.

\section{Gráficos estadísticos: Enseñanza y aprendizaje}

Bajo la misma idea de asumir las tablas estadísticas como formas de representación gráfica de datos, se tienen en cuenta elementos que impactan en el proceso de enseñanzaaprendizaje de los gráficos estadísticos. Entre estos elementos está la necesidad de conocimientos previos, las habilidades que se requieren para la comprensión de los gráficos y los niveles en que se puede ubicar un individuo frente a la lectura de estos. Según Batanero y Godino (2002) algunos aspectos que pueden afectar la comprensión de gráficos estadísticos, se relacionan con conocimientos previos en torno a:

- El tema al que se refiere el gráfico. Si el estudiante no está familiarizado con el contexto de los datos o simplemente los mismos carecen de sentido o importancia para él, la comprensión de la información estadística que se pretende reportar en el respectivo gráfico podrá ser afectada por desconocimiento del contexto.

- El contenido matemático inmerso en el gráfico, esto es, conceptos numéricos (porcentajes, decimales, escalas, etc.), relaciones de orden, operaciones contenidas en el mismo, proporciones, áreas, etc.

- El tipo de gráfico estadístico empleado (barras, histogramas, pictograma, diagrama de cajas, cartogramas, etc.) según el o los tipos de variables estadísticas que se representan o la información que se quiere relacionar en el mismo.

Para la comprensión de los gráficos estadísticos y por ende de la información en ellos reportada, se requiere según Friel, Curcio y Bright (2001) de las habilidades de traducción, interpretación, extrapolación e interpolación (Cuadro 2).

Cuadro 2 - Habilidades para la comprensión de gráficos estadísticos

\begin{tabular}{|c|c|}
\hline Traducción & $\begin{array}{c}\text { Práctica para transitar entre distintos tipos de representación de un mismo conjunto de datos. } \\
\text { Esto es, la habilidad de pasar de tablas de datos en bruto (no ordenados) a tablas de conteo, de } \\
\text { frecuencia o de contingencia. Pasar de tablas estadísticas a gráficos, y poder usar distintos } \\
\text { gráficos estadísticos para representar un mismo conjunto de datos. }\end{array}$ \\
\hline
\end{tabular}


DOI: $10.20396 /$ zet.v28i0.8656553

\begin{tabular}{|c|c|}
\hline Interpretación & $\begin{array}{c}\text { Pericia para establecer relaciones entre los datos (de orden, de contenencia, etc.) y tomar en } \\
\text { cuenta los más representativos para comprender la información que se representa. }\end{array}$ \\
\hline $\begin{array}{c}\text { Extrapolación } \\
\text { /Interpolación }\end{array}$ & $\begin{array}{c}\text { Destreza para identificar tendencias, regularidades o situaciones atípicas presentadas de forma } \\
\text { implícita dentro del conjunto de datos. }\end{array}$ \\
\hline
\end{tabular}

Fuente: Propia inspirada en Friel, Curcio y Bright (2001)

Dependiendo de los conocimientos previos, las habilidades y la construcción conceptual que se esté desarrollando alrededor del objeto de estudio, los individuos pueden ubicarse en un determinado nivel de interpretación de gráficos estadísticos. Siguiendo la propuesta de Friel, Curcio y Bright (2001) se expone en el Cuadro 3 una descripción ampliada de los cuatro niveles.

Cuadro 3 - Niveles de interpretación de gráficos estadísticos

\begin{tabular}{|c|c|c|}
\hline $\begin{array}{c}\text { Nivel 1. } \\
\text { deer los } \\
\text { datos }\end{array}$ & $\begin{array}{c}\text { Se realiza una lectura literal (básica o superficial) del gráfico. No hay una interpretación de la } \\
\text { información contenida en el mismo sino que se identifica expresamente lo que a simple vista } \\
\text { se puede ver (cantidades, etiquetas, frecuencias, variables, valores o categorías para la } \\
\text { variable, título, etc.). }\end{array}$ \\
\hline $\begin{array}{c}\text { Nivel 2. } \\
\text { Leer dentro } \\
\text { de los datos }\end{array}$ & $\begin{array}{c}\text { Se hace una interpretación e integración de los datos presentados en el gráfico. Una lectura } \\
\text { con mayor nivel de profundidad pero solo hasta el punto de establecer relaciones entre los } \\
\text { datos a partir de los conocimientos previos respecto a conceptos y destrezas matemáticas. Se } \\
\text { establecen relaciones de orden entre frecuencias, se reconoce el tipo de variable de estudio y } \\
\text { se identifica algún comportamiento de los datos a partir de medidas de tendencia central que } \\
\text { se reconocen a simple vista (la moda). }\end{array}$ \\
\hline $\begin{array}{c}\text { Neer más } \\
\text { allá de los } \\
\text { datos }\end{array}$ & $\begin{array}{r}\text { Se efectúan predicciones e inferencias respecto a información que no se muestra } \\
\text { directamente en los datos presentados en el gráfico: Formulan hipótesis sobre el futuro o } \\
\text { anterior comportamiento del conjunto de datos. Se logra establecer relaciones entre dos o } \\
\text { más variables estadísticas si es que estas se presentan en los gráficos. }\end{array}$ \\
\hline $\begin{array}{c}\text { Nivel 4. } \\
\text { de los detrás datos }\end{array}$ & $\begin{array}{c}\text { El lector valora la completitud y fiabilidad de los datos. Se cuestiona acerca del origen de los } \\
\text { mismos y la intención de su representación de tal o cual manera. Establece cuestionamientos } \\
\text { que requieren de información sobre otras variables estadísticas asociadas al estudio para darle } \\
\text { sentido y utilidad a los datos representados. }\end{array}$ \\
\hline
\end{tabular}

Fuente: Propia inspirada en Friel, Curcio y Bright (2001)

La conjugación entre la teoría de dificultades, obstáculos y errores, las habilidades para la comprensión de gráficos estadísticos, los niveles de lectura e interpretación de los mismos, y la transferencia de estas perspectivas hacia el constructo tablas de frecuencia, orienta la formulación de los instrumentos de recolección de la información para poder caracterizar de forma particular dificultades y errores propios del objeto de investigación.

\section{Estrategia metodológica de investigación}

La investigación de corte cualitativo descriptivo se lleva a cabo en cuatro fases. En primer lugar se formula un compilado de errores y dificultades para cada uno de los procesos de estudio (construcción e interpretación). Este acopio se da partir de las experiencias de aula vividas durante la práctica educativa en relación con la enseñanza de tablas de frecuencia, y de los referentes teóricos asumidos como marco para la investigación. En el segundo momento, para recoger sistemáticamente las evidencias y que estas se conviertan en insumos 
formales, se construyen dos cuestionarios. Un instrumento destinado a la identificación de errores y dificultades presentados en la construcción de tablas de frecuencia el cual contó con un total de 12 situaciones (en el Apéndice A se presentan algunas de ellas). El otro cuestionario compuesto por 8 situaciones con el propósito de identificar errores y dificultades relacionados con la interpretación de tablas (el Apéndice B da a conocer unas de estas situaciones). Para ambos casos se involucran conjuntos de datos agrupados y no agrupados.

La tercera fase, la gestión, se lleva a cabo en cuatro instituciones educativas localizadas en el departamento de Cundinamarca, Colombia. La elección de dichas instituciones corresponde a que son aquellas en las cuales se presentó la propuesta y fue aceptada por directivos o docentes ${ }^{5}$. Los grados escolares son elegidos porque en su momento ya habían trabajado en el aula el objeto estadístico en mención y por ende sus estudiantes estarían en la capacidad de resolver los cuestionarios. Estas condiciones filtraron los grados $7^{\circ}$ y $9^{\circ}$ de tales entidades puesto que los docentes no dieron el permiso o informaron que los participantes no contaban conocimiento previo sobre el tema. El cuestionario de interpretación se desarrolla en dos colegios con 65 estudiantes de entre 11 y 16 años $\left(6^{\circ}\right.$ u $8^{\circ}$ grado de la educación básica). El cuestionario dirigido a la identificación de errores en la construcción se aplica a 100 estudiantes de tres instituciones disímiles. Los estudiantes cursaban $10^{\circ}$ o $11^{\circ}$ grado (educación media) con edades entre los 15 y 18 años. La implementación de los instrumentos en diferentes instituciones (públicas y privadas, con bachillerato técnico, normalista o académico), grados escolares $\left(6^{\circ}, 8^{\circ}, 10^{\circ}\right.$ y $\left.11^{\circ}\right)$, y a estudiantes con diversas edades (entre 11 y 18 años), permitió contar con respuestas de participantes inmersos en diversos contextos (Cuadro 4) y asociadas a diferentes procesos de enseñanza-aprendizaje.

Cuadro 4 - Caracterización de los participantes

\begin{tabular}{|c|c|c|c|c|}
\hline \multicolumn{2}{|c|}{ ESTUDIANTES } & \multirow{2}{*}{ GRADO } & \multirow{2}{*}{$\begin{array}{c}\text { TIPO DE } \\
\text { INSTITUCIÓN }\end{array}$} & \multirow{2}{*}{$\begin{array}{l}\text { INSTRUMENTO } \\
\text { DILIGENCIADO }\end{array}$} \\
\hline CANTIDAD & EDADES & & & \\
\hline $\begin{array}{c}1 \\
19 \\
17 \\
2\end{array}$ & $\begin{array}{l}15 \text { años } \\
16 \text { años } \\
17 \text { años } \\
18 \text { años }\end{array}$ & $11^{\circ}$ & $\begin{array}{l}\text { Institución pública } \\
\text { municipal: Bachiller } \\
\text { académico }\end{array}$ & \multirow{3}{*}{$\begin{array}{c}\text { Cuestionario 1: } \\
\text { Construcción de tablas de } \\
\text { frecuencia }\end{array}$} \\
\hline $\begin{array}{c}17 \\
11 \\
3 \\
\end{array}$ & $\begin{array}{l}15 \text { años } \\
16 \text { años } \\
17 \text { años } \\
\end{array}$ & $10^{\circ}$ & $\begin{array}{l}\text { Institución pública: } \\
\text { Escuela Normalista }\end{array}$ & \\
\hline $\begin{array}{c}2 \\
23 \\
5\end{array}$ & $\begin{array}{l}15 \text { años } \\
16 \text { años } \\
17 \text { años }\end{array}$ & $11^{\circ}$ & $\begin{array}{l}\text { Institución privada: } \\
\text { Bachiller académico }\end{array}$ & \\
\hline $\begin{array}{c}19 \\
7 \\
3 \\
2\end{array}$ & $\begin{array}{l}11 \text { años } \\
12 \text { años } \\
13 \text { años } \\
14 \text { años }\end{array}$ & $6^{\circ}$ & $\begin{array}{l}\text { Institución pública } \\
\text { municipal: Bachiller } \\
\text { académico }\end{array}$ & $\begin{array}{c}\text { Cuestionario 2: } \\
\text { Interpretación de tablas de } \\
\text { frecuencia }\end{array}$ \\
\hline
\end{tabular}

\footnotetext{
${ }^{5}$ La propuesta se presentó a 10 entidades educativas y solo en cuatro de ellas se abrió el espacio para la gestión de la investigación.
} 
DOI: $10.20396 /$ zet.v28i0.8656553

\begin{tabular}{|c|c|c|c|c|}
\hline 1 & 12 años & & & \\
15 & 13 años & & Institución pública: & \\
11 & 14 años & $8^{\circ}$ & Colegio Técnico & \\
5 & 15 años & & Comercial & \\
2 & 16 años & & & \\
\hline
\end{tabular}

Fuente: Propia

En la fase cuatro se asume el ejercicio metódico de revisar y analizar cada una de las respuestas plasmadas en los cuestionarios, convirtiendo la información en datos. Inicialmente se organizan los datos de acuerdo a la perspectiva de Batanero y Godino (2002) transfiriendo los errores en la construcción de gráficos a la construcción de tablas. Encontrando respuestas (errores) comunes y atendiendo a la experiencia docente respecto a la enseñanza de este objeto, se refina la clasificación de los datos y se generan categorías de análisis. Las categorías permiten tipificar los datos en pro de los elementos constitutivos del objeto de estudio y caracterizar la población frente a los errores y dificultades en la construcción e interpretación de tablas de frecuencia. Por último, una definición profunda y diferenciada de tales categorías, atendiendo a los procesos y los conceptos involucrados en las tablas de frecuencia, conlleva a la consolidación de una taxonomía particular para tal objeto, principal conclusión y aporte teórico de esta investigación al campo de la Educación Estadística.

\section{Análisis y Resultados}

Con respecto a la construcción de tablas, se identifica que los participantes realizaron la tabla de frecuencias con datos no agrupados a pesar de la gran cantidad de datos que tuvieron que manipular. Otros determinaron una amplitud inadecuada para los intervalos y por ende obtuvieron una cantidad muy grande o muy pequeña de estos (determinan intervalos de amplitud muy grande y amplitud diferente), lo que devela que no tuvieron en cuenta la dispersión de los datos. También realizaron cálculos erróneos de las frecuencias y entre las frecuencias (sustracción entre la frecuencia simple de dos valores consecutivos de la variable para completar la columna de frecuencia acumulada); omitieron valores de la variable estadística de estudio (excluyeron o repitieron datos); intercambiaron las frecuencias (v.g. la simple con la acumulada); incluyeron información que aunque estaba expuesta en la situación, no podía ser clasificada como variable de estudio; y repitieron datos en la distribución de frecuencias (Ilustración 1).

Las falencias en conocimientos previos, la lectura parcial o superficial del contexto de la situación, y la falta de representatividad de la amplitud de los intervalos, pueden ser tomadas como dificultades asociadas a los procesos de enseñanza de las matemáticas. Dificultades afines con los conocimientos previos acerca del uso de intervalos en una distribución de frecuencias. Sin embargo, se logra establecer que en ningún caso los estudiantes omitieron el valor de la variable estadística con frecuencia cero, lo cual deja en evidencia que este no es un error común en la construcción de tablas de frecuencia, como se suponía a partir de la experiencia docente. 
DOI: $10.20396 /$ zet.v28i0.8656553

Las actividades de interpretación de la información permiten identificar que los participantes usan de manera indistinta una u otra frecuencia para concluir o dar respuestas. Manifiestan confusión entre frecuencia relativa simple y frecuencia acumulada. Otros afirman no recordar o no comprender el significado de la frecuencia, lo cual los lleva a confundir una determinada frecuencia con el valor de la variable estadística que le corresponde o viceversa. Escogen resultados en pro de su subjetividad y no basados en los datos. Omiten la diferente de amplitud que muestran los intervalos usados en la tabla. Confunden mayor valor de la variable con mayor valor de la frecuencia. Usan un extremo del intervalo como representante del mismo. Estos y otros errores se pueden visualizar en la Ilustración 2.

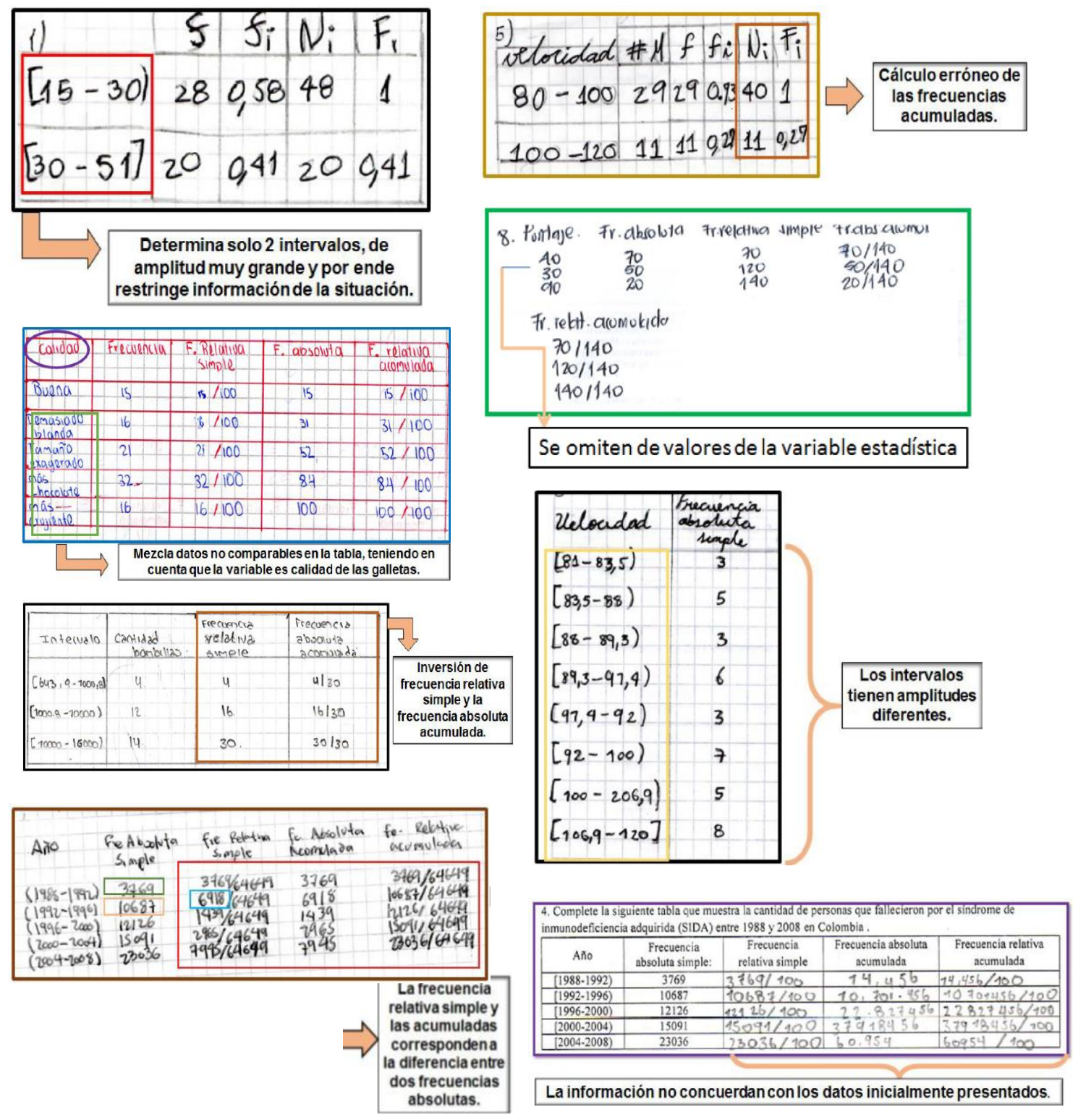

Ilustración 1 - Evidencias de errores en la construcción de tablas de frecuencia

Fuente: Producciones de los participantes de la investigación 
DOI: $10.20396 /$ zet.v28i0.8656553

La mayoría de los participantes limitan el análisis/interpretación de los datos reportados en las tablas de frecuencia debido a una visualización superficial o parcial de la información consignada en ellas. Solo dan respuesta siempre y cuando las preguntas indaguen por algún dato en particular expuesto explícitamente (lo puedan identificar con una verificación visual) en alguna de las celdas. Esto devela poca habilidad para realizar una visualización general de un conjunto de datos y por tanto la falta de destreza para efectuar predicciones e inferencias a partir de los datos y el análisis en conjunto de los mismos.
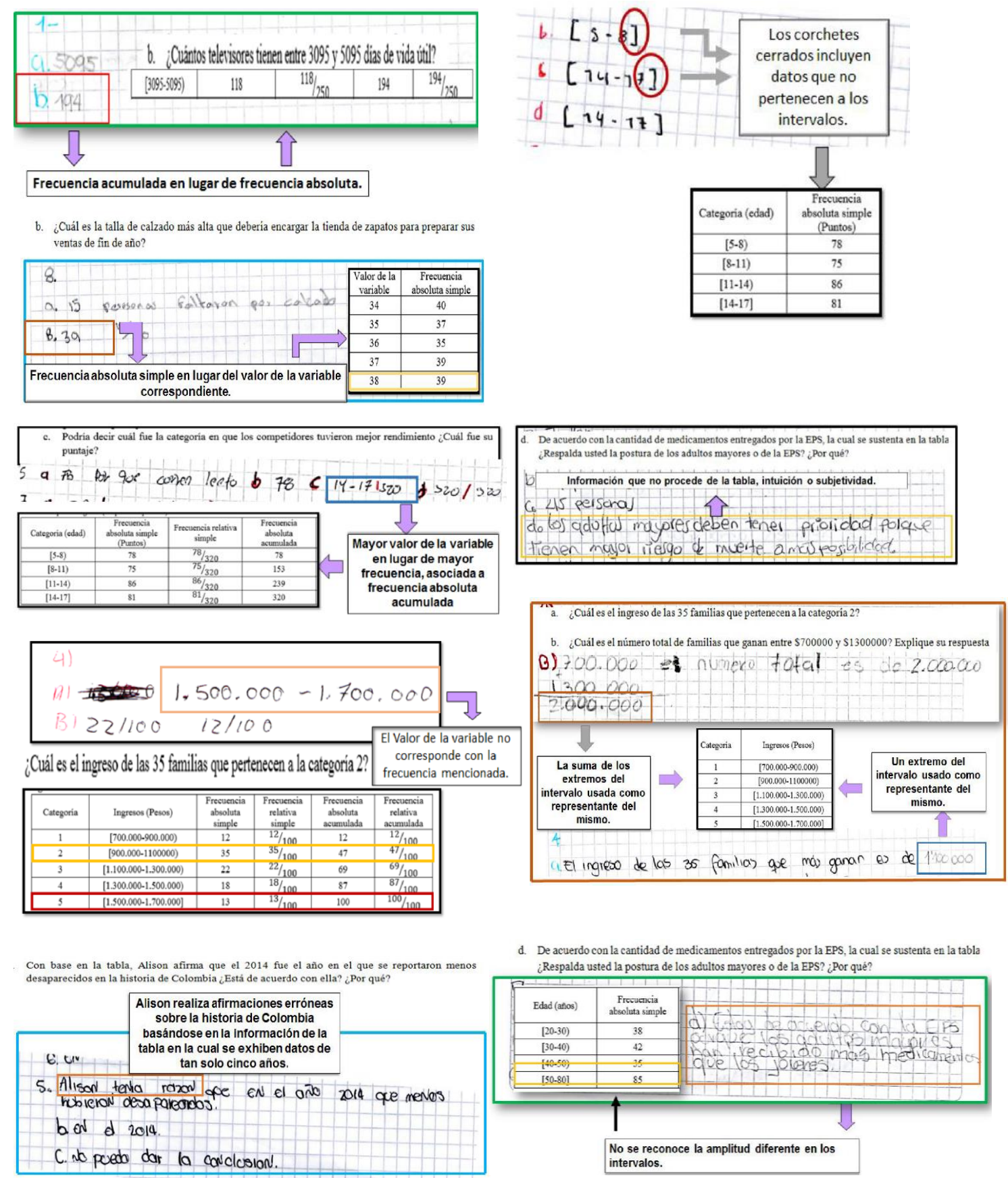

Ilustración 2 - Evidencias de errores en la interpretación de tablas de frecuencia Fuente: Producciones de los participantes de la investigación 
De otra parte se evidencia que los participantes no reconocen, y por ende no valoran, la información que aporta la frecuencia absoluta acumulada. Por ejemplo, cuando se cuestiona respecto a una frecuencia absoluta acumulada expuesta explícitamente en la tabla, los estudiantes repiten el proceso algorítmico (realizan la suma de las frecuencias absolutas simples correspondientes) dejando de lado la representatividad de la información que ya ofrece la tabla. Estos errores se vinculan con el desconocimiento de los tipos de frecuencia (absoluta, relativa, acumulada) y su significado, y son producto de las dificultades asociadas a los procesos de enseñanza de las matemáticas. En particular se observan falencias en conocimientos previos, pero también ansiedad de terminar una tarea. Dificultades vinculadas a afectividades o emociones hacia las matemáticas.

Una vez se analizan todos los datos se consigue sintetizar en las Gráfica 1 y Gráfica 2 la frecuencia con la que ocurre cada error según el proceso (construcción o interpretación). Sin importar el número de participantes que desarrolló las actividades, lo que se cuantifica es la cantidad de veces que aparece el error en la totalidad de los instrumentos. Así, el número de veces que se exhibe el error es mayor que la cantidad de estudiantes participantes. Pudo ser que el error se repitiera varias veces en una o varias actividades, o en una misma actividad se presentaran diferentes errores. La frecuencia del error se contrasta con el total de repuestas en las que este se podía identificar.

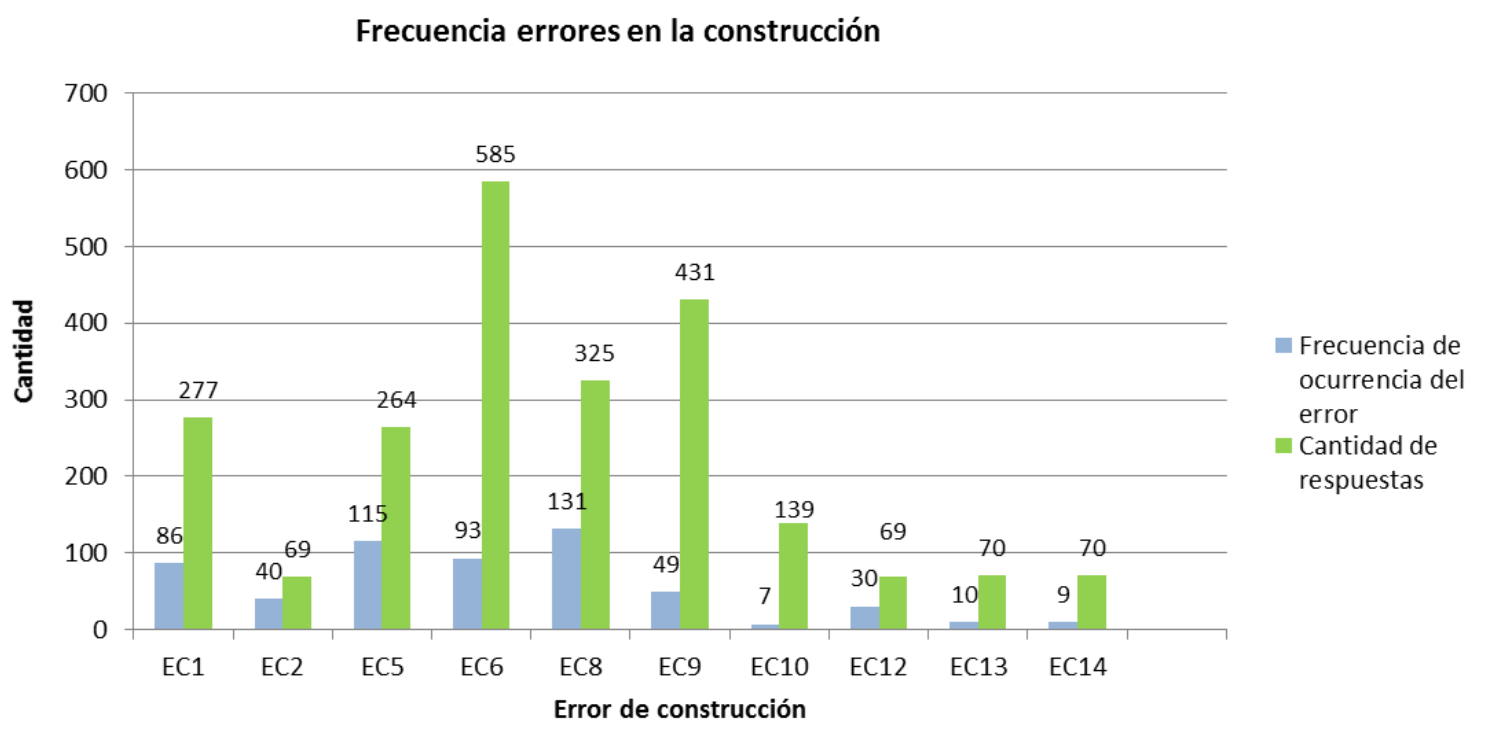

Gráfica 1 - Ocurrencia de errores en la construcción de tablas de frecuencia Fuente: Propia

En la Gráfica 1 se puede reconocer que para el primer error de construcción [EC1] se tuvo un total de 86 evidencias de su existencia, dentro de un total de 277 respuestas. Respuestas de todos los participantes y que estaban asociadas a las situaciones en las que se podía cometer el error. En la Gráfica 2 se contempla que para las situaciones que abordan el primer error de interpretación [EI1] se tuvo un total de 271 respuestas y de ellas 74 daban muestra de la existencia del error. 
DOI: $10.20396 /$ zet.v28i0.8656553

Frecuencia de errores en la interpretacióm

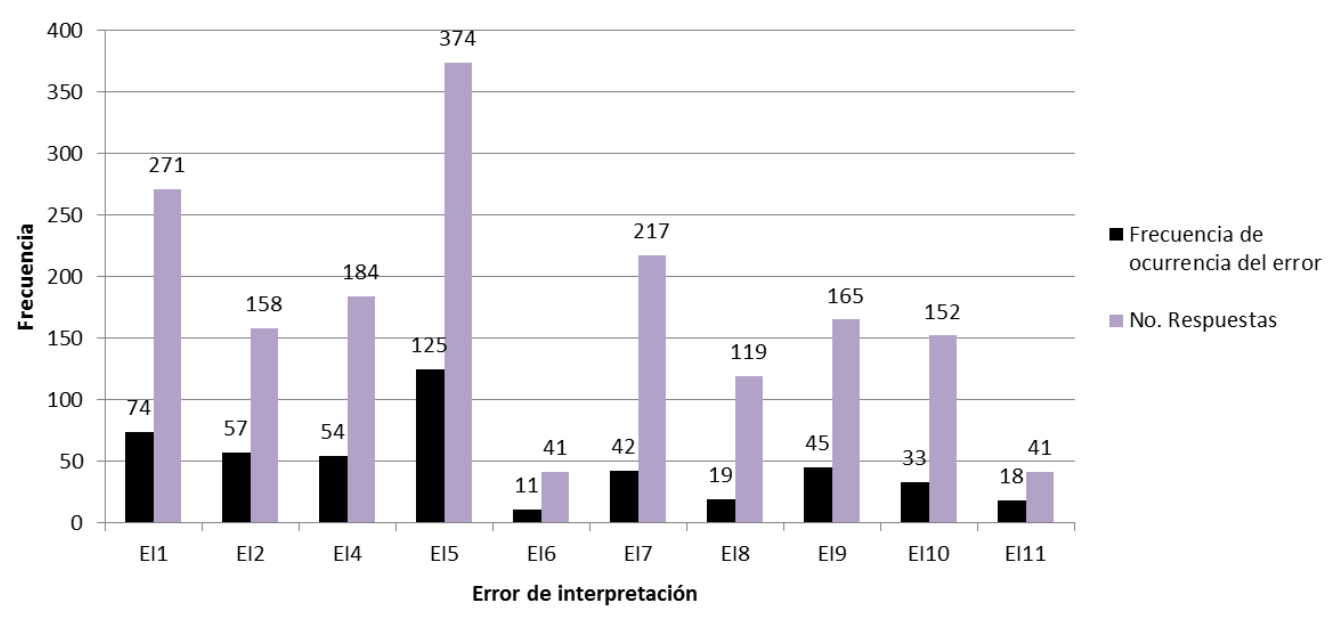

Gráfica 2 - Ocurrencia de errores en la interpretación de tablas de frecuencia Fuente: Propia

Acopiadas, organizadas y analizadas las evidencias se logra consolidar una taxonomía que permite caracterizar los errores y las potenciales dificultades que los generan (Cuadro 5). Se listan no solo aquellos errores evidenciados con los instrumentos, sino algunos otros que se consideran importantes y que fueron identificados durante las prácticas docentes de las autoras, pero que no tuvieron resonancia en la indagación, aunque se contemplaron para que emergieran a través de las situaciones propuestas.

Cuadro 5 - Taxonomía de errores y dificultades en la construcción e interpretación de tablas de frecuencia

\begin{tabular}{|c|c|}
\hline \multicolumn{2}{|c|}{ PROCESO DE CONSTRUCCIÓN } \\
\hline ERRORES & $\begin{array}{l}\text { DIFICULTADES } \\
\end{array}$ \\
\hline $\begin{array}{l}\text { EC }_{1} \text {. Ignorar la dispersión de los datos, eligiendo de } \\
\text { forma inadecuada la amplitud de los intervalos. } \\
\text { Proporciona demasiados o pocos intervalos cuando } \\
\text { trabaja con datos agrupados. }\end{array}$ & $\begin{array}{l}\mathrm{DC}_{1} . \text { Omitir la representatividad de los datos } \\
\text { estadísticos. }\end{array}$ \\
\hline $\begin{array}{l}\text { EC }_{2} \text { Mezclar datos que no son comparables ( } v . g \text {. } \\
\text { incluir en la tabla valores que la variable estadística no } \\
\text { puede tomar por su naturaleza cualitativa o } \\
\text { cuantitativa). }\end{array}$ & $\begin{array}{l}\mathrm{DC}_{2} \text {.Ignorar la coherencia entre la situación en la que } \\
\text { se enmarca los datos estadísticos y lo valores de la } \\
\text { variable estadística. }\end{array}$ \\
\hline $\begin{array}{l}\mathrm{EC}_{3} \text {.Invertir los elementos involucrados en el } \\
\text { algoritmo para calcular la frecuencia relativa simple o } \\
\text { acumulada (numerador-denominador). }\end{array}$ & $\begin{array}{l}\mathrm{DC}_{3} . \text { Presentar confusión en cuanto a la definición de } \\
\text { frecuencia relativa como relación parte-todo o en el } \\
\text { algoritmo de la división. }\end{array}$ \\
\hline $\begin{array}{l}\mathrm{EC}_{4} \text {. En el caso de datos agrupados, omitir un intervalo } \\
\text { por tener frecuencia cero. }\end{array}$ & $\begin{array}{l}\text { DC }_{4} \text { Presentar confusión frente el cero como valor } \\
\text { representativo de la frecuencia de un subconjunto de } \\
\text { datos y su significado como ausencia del valor de la } \\
\text { variable. }\end{array}$ \\
\hline $\begin{array}{l}\text { EC } 5 \text {.Determinar intervalos con amplitudes diferentes } \\
\text { en una misma distribución de frecuencias de datos } \\
\text { agrupados. }\end{array}$ & $\begin{array}{l}\text { DC 5.Ignorar la igualdad entre las longitudes de } \\
\text { intervalos. }\end{array}$ \\
\hline $\begin{array}{l}\text { EC }_{6} \text {. Invertir los tipos de frecuencias en la construcción } \\
\text { de la tabla ( } v . g \text {. ubicar las frecuencias relativas simples }\end{array}$ & $\mathrm{DC}_{6}$. Desconocer los tipos de frecuencia. \\
\hline
\end{tabular}


DOI: $10.20396 /$ zet.v28i0.8656553

\begin{tabular}{|c|c|}
\hline \multicolumn{2}{|l|}{ en la columna de las frecuencias absolutas simples). } \\
\hline $\begin{array}{l}\text { EC }_{7} \text {.Invertir los valores de una variable estadística con } \\
\text { sus respectivas frecuencias. }\end{array}$ & 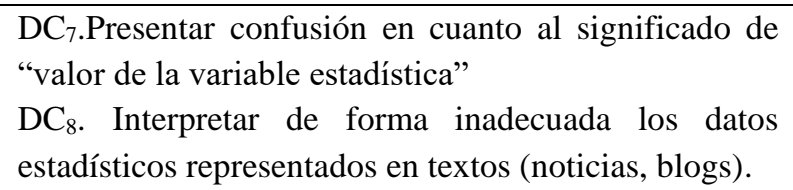 \\
\hline $\begin{array}{l}\text { EC }_{8} \text { Excluir o repetir datos en la construcción de tablas } \\
\text { de frecuencia de conjuntos de datos agrupados. }\end{array}$ & $\begin{array}{l}\text { DC } 9 . \text { Usar de forma inadecuada intervalos abiertos o } \\
\text { cerrados. }\end{array}$ \\
\hline $\begin{array}{llll}\mathrm{EC}_{9} \text { Calcular } & \text { la } & \text { frecuencia } & \text { acumulada } \\
\text { incorrectamente. } & & & \end{array}$ & $\mathrm{DC}_{10}$.Presentar confusión en el algoritmo de la suma. \\
\hline $\begin{array}{l}\mathrm{EC}_{10} \text {.Omitir valores de la variable estadística al } \\
\text { construir tablas de frecuencia. }\end{array}$ & $\begin{array}{l}\mathrm{DC}_{11} \text { Interpretar de forma inadecuada la lectura de los } \\
\text { datos estadísticos desde la representación verbal. }\end{array}$ \\
\hline $\begin{array}{l}\mathrm{EC}_{11} \text {. Invertir las frecuencias de dos o más valores de la } \\
\text { variable estadística. }\end{array}$ & $\begin{array}{l}\mathrm{DC}_{12} \text {. No tener en cuenta la correspondencia entre el } \\
\text { conteo de datos y el valor de la variable estadística a } \\
\text { la que corresponde la frecuencia. }\end{array}$ \\
\hline $\begin{array}{l}\mathrm{EC}_{12} \text {.Incluir percepciones personales sin atender la } \\
\text { información estadística proveniente de la situación. } \\
\text { EC }_{13} \text {. Construir tablas de frecuencia que no concuerdan } \\
\text { con la información y el contexto de la situación. }\end{array}$ & $\begin{array}{l}\mathrm{DC}_{13} \text {.Ignorar u omitir información de la situación } \\
\text { priorizando la subjetividad. }\end{array}$ \\
\hline $\begin{array}{l}\mathrm{EC}_{14} . \text { Determinar la frecuencia acumulada a partir de la } \\
\text { diferencia entre frecuencias. }\end{array}$ & $\begin{array}{l}\text { DC }_{14 .} \text { Desconocer el significado de frecuencia } \\
\text { acumulada. }\end{array}$ \\
\hline \multicolumn{2}{|c|}{ PROCESO DE INTERPRETACIÓN } \\
\hline $\begin{array}{l}\text { EI }_{1} \text {.Proporcionar la frecuencia del valor de la variable } \\
\text { que no corresponde con el tipo de frecuencia } \\
\text { solicitada. }\end{array}$ & $\begin{array}{l}\text { DI }_{1} \text {. Desconocer los tipos de frecuencia (absoluta, } \\
\text { relativa, acumulada). }\end{array}$ \\
\hline $\begin{array}{l}\mathrm{EI}_{2} \text {. Establecer relaciones inconsistentes entre las } \\
\text { frecuencias de dos o más valores de la variable } \\
\text { estadística. }\end{array}$ & $\begin{array}{l}\mathrm{DI}_{2} \text {. Desconocer las relaciones numéricas entre dos o } \\
\text { más frecuencias. }\end{array}$ \\
\hline $\begin{array}{l}\mathrm{EI}_{3} \text {. Establecer de forma incomprensible las tendencias } \\
\text { en un conjunto de datos a través de la información } \\
\text { presentada en la tabla de frecuencias. }\end{array}$ & $\begin{array}{l}\mathrm{DI}_{3} \text {. Visualizar parcialmente o de manera aislada el } \\
\text { conjunto de datos estadísticos. }\end{array}$ \\
\hline $\begin{array}{l}\text { EI } 4 \text {.Asociar el valor de la variable estadística } \\
\text { cuantitativa con una de las frecuencias proporcionadas } \\
\text { en la tabla que no le corresponde, o viceversa. }\end{array}$ & $\begin{array}{l}\mathrm{DI}_{4} \text {. Desatender a convenios para la interpretación de } \\
\text { tablas estadísticas. Lectura horizontal de las filas de } \\
\text { las tablas, dado que el tipo de frecuencia } \\
\text { proporcionada concuerda con la solicitada pese a no } \\
\text { ser el valor correspondiente. }\end{array}$ \\
\hline $\begin{array}{l}\text { EI } 5 . \text { Concluir información que no concuerda con los } \\
\text { datos estadísticos proporcionados en la tabla de } \\
\text { frecuencias. }\end{array}$ & $\begin{array}{l}\text { DI5.Inducir apresuradamente, como intento de } \\
\text { generalización de la información. }\end{array}$ \\
\hline EI $_{6 . \text { Ignorar la dispersión de los datos estadísticos. }}$ & $\mathrm{DI}_{6}$. Omitir la representatividad de los datos. \\
\hline $\begin{array}{l}\text { EI } 7 . \text { Intercambiar los valores de una variable estadística } \\
\text { cuantitativa con los valores de sus frecuencias. }\end{array}$ & $\begin{array}{l}\text { DI. Presentar confusión respecto al significado de } \\
\text { "valor de la variable cuantitativa" versos frecuencia } \\
\text { del valor de la variable. }\end{array}$ \\
\hline $\begin{array}{l}\text { EI } . \text { Identificar el valor de la variable solicitado, sin } \\
\text { embargo, en el caso de datos agrupados, incluye o } \\
\text { excluye valores del mismo. }\end{array}$ & $\begin{array}{l}\text { DI }_{8} \text { Interpretar de forma inadecuada intervalos } \\
\text { abiertos o cerrados en representaciones tabulares de } \\
\text { datos agrupados. }\end{array}$ \\
\hline $\begin{array}{l}\text { EI } 9 \text {.Elegir como valor de la variable cuantitativa, un } \\
\text { representante del intervalo ( } v . g \text {. el valor inicial, el } \\
\text { final, la diferencia o la suma de los extremos). }\end{array}$ & $\begin{array}{l}\text { DI } 9 \text {.Desconocer el significado de valor de la variable } \\
\text { estadística. }\end{array}$ \\
\hline $\mathrm{EI}_{10}$.Confundir, en el caso de la variable cuantitativa, & $\mathrm{DI}_{10}$. Desconocer o confundir el significado de valor \\
\hline
\end{tabular}


DOI: $10.20396 /$ zet.v28i0.8656553

\begin{tabular}{|l|l|}
\hline $\begin{array}{l}\text { el mayor valor de la variable con la mayor frecuencia, } \\
\text { o viceversa. }\end{array}$ & $\begin{array}{l}\text { de la variable estadística cuantitativa y frecuencia } \\
\text { absoluta. }\end{array}$ \\
\hline $\begin{array}{l}\text { EI }_{11} \text {.Concluir información a partir de percepciones } \\
\text { personales sin tener en cuenta los datos } \\
\text { proporcionados en la tabla. }\end{array}$ & $\begin{array}{l}\text { DI } \\
\text { tabla o en la situación priorizando la subjetividad. }\end{array}$ \\
\hline
\end{tabular}

Fuente: Elaboración propia

Los resultados generales se consolidan en los Gráficos 3 y 4. En ellos, además de exhibirse el total de respuestas obtenidas para cada situación, se representa la frecuencia de ocurrencia de cada error por cada una de las situaciones. A partir de estos datos es posible establecer una razón entre el número de respuestas obtenidas y la cantidad de ellas en las que se presentan o no errores.

Por ejemplo, en el Gráfico 3 para la Situación 12 se acopiaron 80 respuestas, de las cuales en 12 se identificó el error [EC6] y en 68 no hubo error alguno. Para la Situación 1 se observa que de las 73 respuestas acopiadas, solamente 11 son correctas. De esto se infiere que 62 respuestas fueron incorrectas, pero se puede calcular que hubo un total de 75 errores

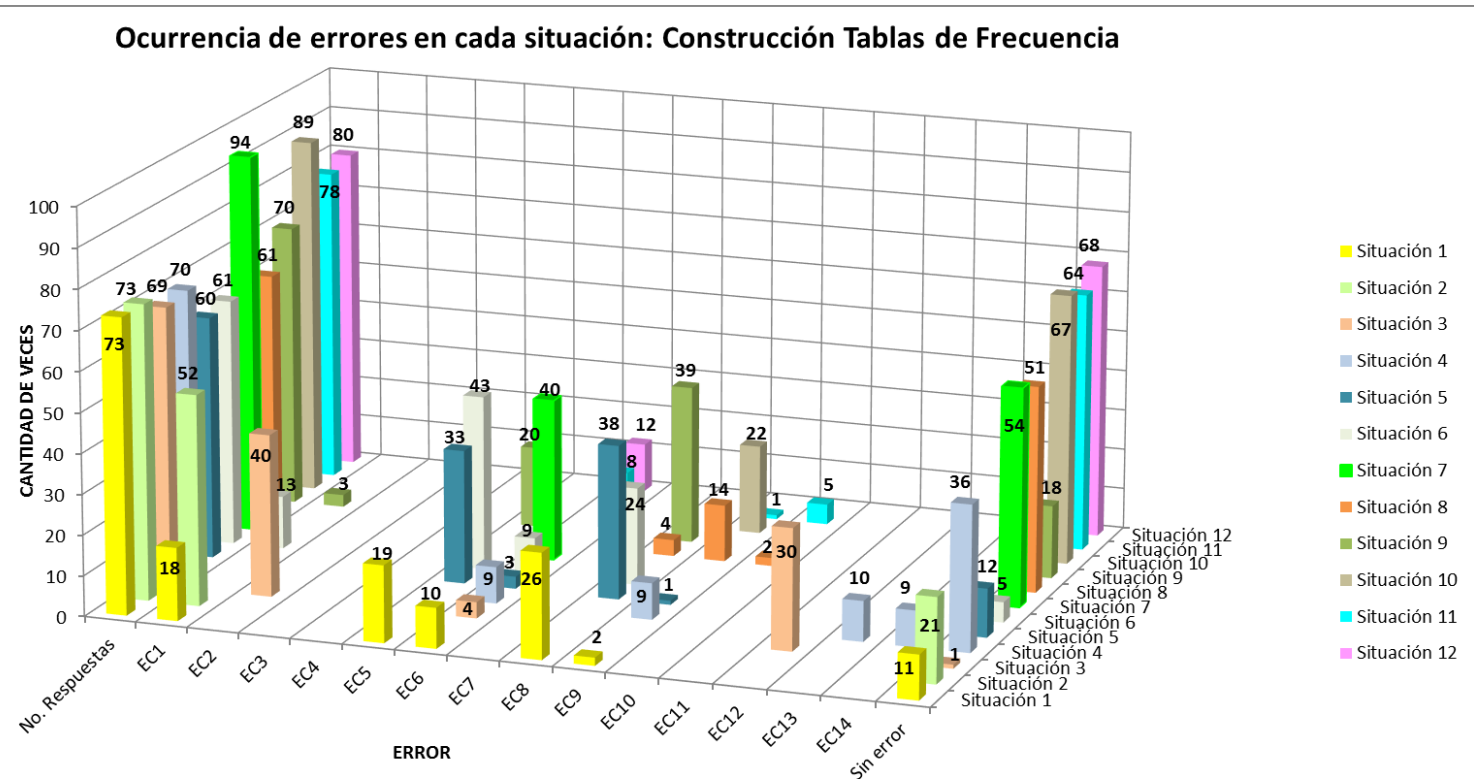

$\left(\mathrm{EC}_{1}, \mathrm{EC}_{5}, \mathrm{EC}_{6}, \mathrm{EC}_{8} \mathrm{y} \mathrm{EC}_{9}\right)$, lo que indica que un mismo error se presentó más de una vez en esta situación.

Gráfica 3 - Ocurrencia de errores en cada situación: Construcción tablas de frecuencia Fuente: Propia

Con base en estos datos se confirma la existencia de errores relacionados de manera particular con la construcción de tablas de frecuencia. Los errores no son los mismos que la literatura viene reportando para la construcción de gráficos estadísticos. En los errores y sus respectivas dificultades se develan elementos particulares y conceptos constitutivos propios del objeto tablas de frecuencia. Estos resultados llevan a hacer un llamado a los educadores estadísticos para que tengan en cuenta la Taxonomía expuesta en el Cuadro 5 y a partir de ella generar estrategias que permitan sortear tales dificultades y por ende evitar o superar los errores reportados en la presente investigación. 


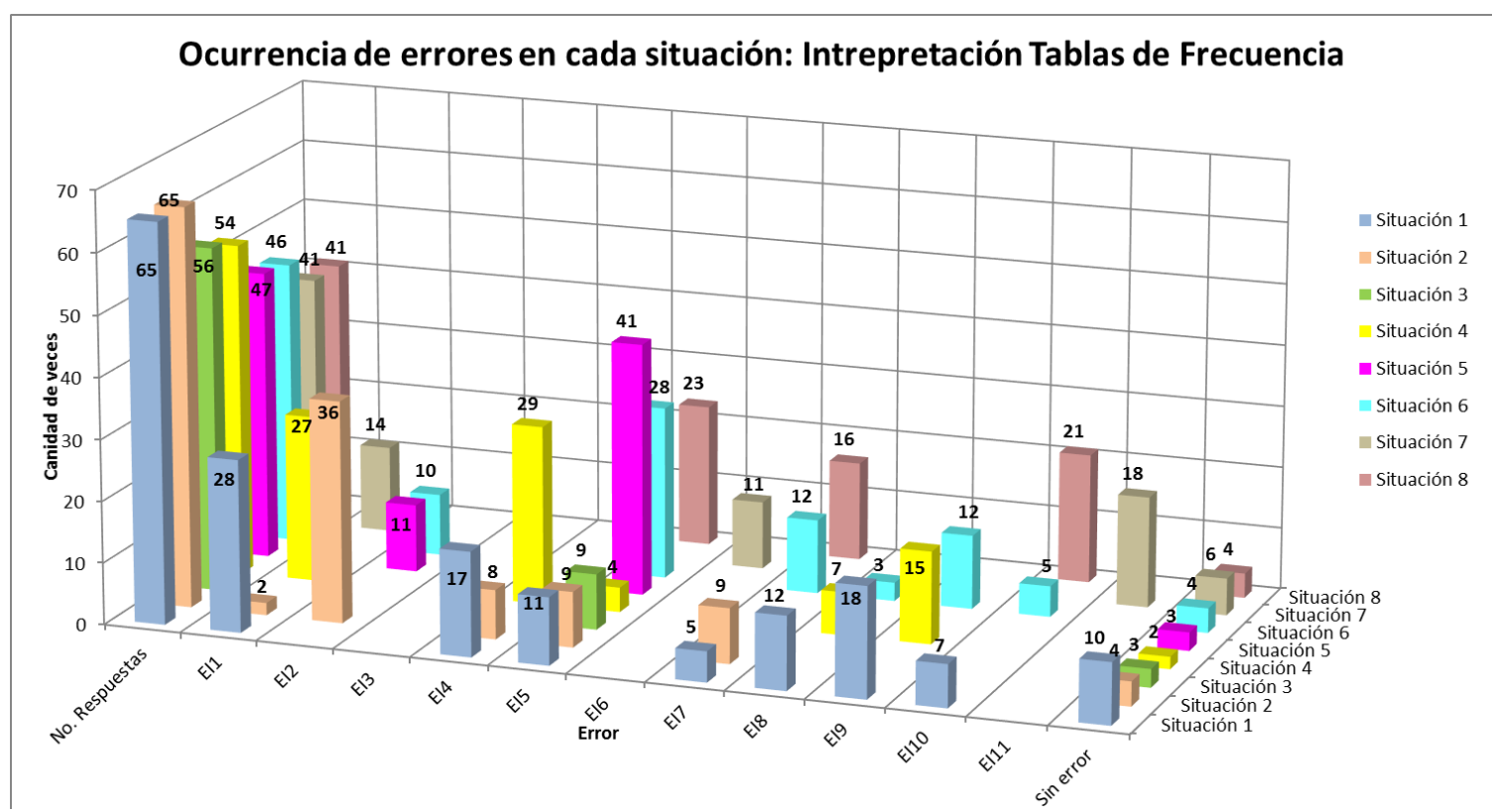

Gráfica 4 - Ocurrencia de errores en cada situación: Interpretación tablas de frecuencia Fuente: Propia

Uno de los puntos fuertes de la investigación es el potencial de los datos para poder caracterizar detalladamente a los participantes alrededor del objetivo propuesto. Así, con respecto a los errores que se comenten en la interpretación de tablas de frecuencia, se identifica que la mayoría de los estudiantes incurre de manera reiterada en el error $\mathrm{EI}_{5}$, esto es, concluir información que no concuerda con los datos expuestos en la tabla. En el caso de los datos agrupados son errores recurrentes tanto el $\mathrm{EI}_{8}$, identificar el valor de la variable solicitado, sin embargo, en el caso de datos agrupados, incluye o excluye valores del mismo; como el EI ${ }_{9}$, elegir como valor de la variable cuantitativa, un representante del intervalo (v.g. el valor inicial, el final, la diferencia o la suma de los extremos).

Tomando como referencia los análisis realizados a cada una de las respuestas acopiadas, se resalta que la mayor parte de las dificultades identificadas son de un mismo origen. En el Cuadro 6 se puede observar que las dificultades están asociadas a los procesos de enseñanza de las matemáticas. De manera específica las dificultades se relacionan con falencias respecto a los conocimientos previos acerca del uso e interpretación de los intervalos en una tabla de frecuencias. Esto se puede afirmar ya que todos los estudiantes presentaron al menos una vez el error $\mathrm{EC}_{5}$ (determinar intervalos con amplitudes diferentes) o el error $\mathrm{EC}_{8}$ (excluir o repetir datos en la construcción de la tabla). Estos resultados sugieren la necesidad de profundizar durante el proceso educativo en la construcción de tablas de frecuencia de datos agrupados, haciendo explícitos sus componentes y la relación entre cada uno de ellos, especialmente lo referido a los intervalos, y la diferencia entre frecuencia, valor de la variable y dato representativo. 
DOI: $10.20396 /$ zet.v28i0.8656553

Cuadro 6 - Tipo de dificultades en la construcción e interpretación de tablas de frecuencia

\begin{tabular}{|c|c|}
\hline $\begin{array}{l}\text { DIFICULTADES EN LA } \\
\text { CONSTRUCCIÓN [DC }] \text { E } \\
\text { INTERPRETACIÓN [DI }]\end{array}$ & $\begin{array}{l}\text { TIPO DE DIFICULTAD } \\
\text { (Socas, 1997) }\end{array}$ \\
\hline $\begin{array}{l}\mathrm{DC}_{1}, \mathrm{DC}_{2}, \mathrm{DC}_{3}, \mathrm{DC}_{4}, \mathrm{DC}_{6}, \mathrm{DC}_{7}, \mathrm{DC}_{8}, \mathrm{DC}_{9}, \\
\mathrm{DC}_{10}, \mathrm{DC}_{11}, \mathrm{DC}_{12}, \mathrm{DC}_{13}, \mathrm{DC}_{12}, \mathrm{DC}_{13} . \\
\mathrm{DI}_{1}, \mathrm{DI}_{2}, \mathrm{DI}_{3}, \mathrm{DI}_{4}, \mathrm{DI}_{5}, \mathrm{DI}_{6}, \mathrm{DI}_{8}, \mathrm{DI}_{9}, \mathrm{DI}_{10}\end{array}$ & $\begin{array}{l}\text { Asociadas a los procesos de enseñanza desarrollados } \\
\text { para el aprendizaje de las matemáticas: en particular } \\
\text { relacionadas con los conocimientos previos. }\end{array}$ \\
\hline $\begin{array}{l}\mathrm{DC}_{5} \\
\mathrm{DI}_{7}\end{array}$ & $\begin{array}{l}\text { Asociada a la complejidad del objeto en matemáticas: } \\
\text { Variedad de significados. }\end{array}$ \\
\hline $\begin{array}{l}\mathrm{DC}_{8}, \mathrm{DC}_{9}, \mathrm{DC}_{13}, \mathrm{DEC}_{12} \\
\mathrm{DI}_{4}, \mathrm{DI}_{5}, \mathrm{DEI}_{11}\end{array}$ & $\begin{array}{l}\text { Asociada a actitudes afectivas y emocionales hacia } \\
\text { las matemáticas: Ansiedad por acabar una tarea. }\end{array}$ \\
\hline
\end{tabular}

Fuente: Propia

A parte de los resultados propios asociados al objetivo de estudio (la Taxonomía y la caracterización de los participantes), uno de los valores agregados que deja la investigación es el contar con un compilado de situaciones enunciadas en los dos cuestionarios. Esto es, los instrumentos de recolección de la información se convierten en herramientas para los docentes. Se pueden replicar con el fin de caracterizar diversas poblaciones escolares y a partir de los resultados se invita a refinar los instrumentos, y precisar o ampliar la Taxonomía, buscando con ello el enriquecimiento del aporte teórico de la presente investigación.

\section{Conclusiones}

Se reconoce que la información acopiada, dado que es recogida en diversas instituciones educativas y distintos grados de escolaridad, es fundamento suficiente para una caracterización completa y amplia de los errores y sus respectivas dificultades. Asunto que permite afirmar que la ocurrencia de los errores es independiente de los procesos de pedagógicos desarrollados en una u otra institución.

Puede precisarse que tanto los errores como las dificultades inherentes al objeto estadístico de estudio y los procesos asociados, se ven condicionados principalmente por falencias en cuanto a conocimientos previos del estudiante, el contexto proporcionado en las situaciones, y por la complejidad propia del constructo tablas de frecuencia. Lo que revela una estrecha relación entre dificultad, obstáculo y error, dejando como potencial camino a recorrer la caracterización de los obstáculos que generan los errores aquí documentados y la tipología de tales obstáculos.

En concordancia con varias de las fuentes citadas en el marco de referencia, se reconocen y aceptan las tablas de frecuencia como una de las principales formas de organizar, resumir y representar datos. Sin embargo, se observa que estas no conservan la misma naturaleza que los gráficos estadísticos, a pesar de que comparten elementos conceptuales (variables estadísticas y su tipo, valor o categorías de la variable, frecuencias, etc.).

Es evidente que los errores y las dificultades abordan constructos estructurales particulares y propios de las tablas como es el caso de la definición y construcción de 
DOI: $10.20396 /$ zet.v28i0.8656553

intervalos para agrupar datos; la diferenciación entre frecuencia y valor de la variable; los cálculos aritméticos para determinar uno u otro tipo de frecuencias; los diferentes tipos de frecuencia y su utilidad en el análisis de los datos, etc. Esto permite afirmar que los errores que se presentan en la enseñanza-aprendizaje de los gráficos estadísticos no son transferibles de manera directa e indistinta a las tablas de frecuencia. No obstante, queda como hipótesis a corroborar, si los errores reportados en la construcción de gráficos son consecuencia de una construcción o interpretación incorrecta de las tablas de frecuencia que sirven de base para su creación. ¿Los errores en la construcción e interpretación de tablas de frecuencia pasan a la categoría de obstáculo en el momento de la construcción y lectura de gráficos estadísticos?

Ya que se logró observar un alto índice en la existencia de errores, y en consecuencia, de dificultades tanto en la construcción como en la interpretación de tablas de frecuencia, se deja en evidencia la inminente necesidad de implementar planes de formación estratégicos entorno a la estructura conceptual (tablas de frecuencia). Asunto de vital importancia en el proceso estadístico de organización y representación de la información. Se busca así apoyar la formación de ciudadanos estadísticamente cultos desde los procesos de interpretación bajo una lectura crítica de tablas de frecuencia y asimismo frente a la organización y representación de datos haciendo uso de estas.

\section{Agradecimientos}

A la Universidad Pedagógica Nacional, Colombia y en particular al Departamento de Matemáticas por su asesoría en el proceso de investigación. Al Semillero de Investigación en Educación Estadística por el acompañamiento y apoyo en la proyección del presente artículo.

\section{Referencias}

Bachelard, G. (1988). La formación del espíritu científico. México: Siglo XXI.

Batanero, C. (2001). Didáctica de la Estadística. Departamento de Didáctica de la Matemática. Facultad de Ciencias de la Educación. Universidad de Granada. Granada, España.

Batanero, C., \& Godino, J. (2002). Estocástica y su Didáctica para maestros. Granada, España: Universidad de Granada, Departamento de Didáctica de la Matemática.

Friel, S., Curcio, F., \& Bright, G. (2001). Making sense of graphs: Critical factors influencing comprehension and instructional implications. Journal for Research in Mathematics Education, 32(2), 124-158.

Ministerio de Educación Nacional (MEN). (1998). Lineamientos Curriculares de Matemáticas. MEN. Bogotá D.C., Colombia.

Ministerio de Educación Nacional (MEN). (2006). Estándares Básicos de competencias en Matemáticas. MEN. Bogotá D.C., Colombia.

Rodríguez, F., \& Sandoval, P. (2012). Habilidades de codificación y decodificación de gráficos estadísticos: Un estudio comparativo en profesores y alumnos de pedagogía en enseñanza básica. Revista da Avaliação da Educação Superior. 17(2), 207-235. 
Socas, M. (1997). Dificultades, obstáculos y errores en el aprendizaje de las matemáticas en la educación secundaria. En L, Rico (Ed.), La Educación Matemática en la Enseñanza Secundaria (pp. 125-154). Barcelona, España: Horsori.

Tauber, L. (2010). Análisis de elementos básicos de alfabetización estadística en tareas de interpretación de gráficos y tablas descriptivas. Ciencias Económicas, (1), 53-74.

\section{APENDICE A. Fragmento de cuestionario para identificación de errores en construcción de tablas de frecuencias}

1. En un estudio se pretende identificar a qué edad suelen presentarse mayores accidentes en moto, para ello se encuestaron 48 motociclistas. Aquí se muestra la información recopilada.

\begin{tabular}{|l|l|l|l|l|l|l|l|l|l|l|l|}
\hline 16 & 28 & 26 & 15 & 33 & 18 & 16 & 30 & 22 & 27 & 21 & 42 \\
\hline 19 & 46 & 34 & 23 & 40 & 16 & 32 & 27 & 25 & 40 & 30 & 37 \\
\hline 41 & 21 & 22 & 30 & 45 & 51 & 26 & 48 & 18 & 39 & 50 & 36 \\
\hline 47 & 48 & 20 & 22 & 47 & 19 & 16 & 51 & 20 & 17 & 27 & 32 \\
\hline
\end{tabular}

Construya la tabla de distribución de frecuencia absoluta que represente la información presentada anteriormente usando intervalos.

2. Una compañía de galletas desea saber la calidad (buenas, muy buenas regulares o malas) de unas galletas que recién salieron al mercado y el gusto de la población hacia ellas para determinar su continuidad; para ello se ofrecieron muestras gratis a la salida de un supermercado y se realizó una encuesta a quienes recibían la muestra. Cinco horas después se recopiló la información obtenida, que se muestra a continuación:

- 15 personas la clasificaron como buena

- 16 personas dijeron que era demasiado blanda

- 21 personas concluyeron que su tamaño era exagerado

- 32 personas dijeron que tenía buen sabor pero en su opinión podría agregarse más chocolate

- 16 personas la clasificaron en muy buena, aunque podría ser más crujiente

Construya la tabla de frecuencias que representa la información suministrada por las personas encuestadas, en relación con la calidad de las galletas.

3. Complete la siguiente tabla que muestra la cantidad de personas que fallecieron por el síndrome de inmunodeficiencia adquirida (SIDA) entre 1988 y 2008 en Colombia.

\begin{tabular}{|c|c|c|c|c|}
\hline Año & $\begin{array}{c}\text { Frecuencia } \\
\text { absoluta simple: }\end{array}$ & $\begin{array}{c}\text { Frecuencia } \\
\text { relativa simple }\end{array}$ & $\begin{array}{c}\text { Frecuencia absoluta } \\
\text { acumulada }\end{array}$ & $\begin{array}{c}\text { Frecuencia relativa } \\
\text { acumulada }\end{array}$ \\
\hline$[1988-1992)$ & 3769 & & & \\
\hline$[1992-1996)$ & 10687 & & & \\
\hline$[1996-2000)$ & 12126 & & & \\
\hline$[2000-2004)$ & 15091 & & & \\
\hline$[2004-2008]$ & 23036 & & & \\
\hline
\end{tabular}

4. En una estación de policía se tiene el siguiente reporte de las velocidades a las que iban 40 conductores multados por exceso de velocidad, en zonas donde el máximo permitido es $79 \mathrm{~km} / \mathrm{h}$. 


\begin{tabular}{|c|c|c|c|c|c|c|c|c|c|}
\hline 120 & 90 & 90 & 91 & 92 & 91,4 & 89,3 & 92 & 83 & 107 \\
\hline 88,9 & 106,9 & 92 & 85 & 87 & 88,3 & 104,3 & 90 & 90 & 82 \\
\hline 83,5 & 87 & 95 & 96 & 119,3 & 100 & 99 & 88 & 91,8 & 115,7 \\
\hline 91,5 & 120 & 85 & 100 & 102,7 & 92 & 116 & 80 & 103,6 & 116 \\
\hline
\end{tabular}

Construya la tabla de distribución de frecuencia absoluta que represente la información respecto a las velocidades a las que se multaron los conductores, usando 8 intervalos.

5. En una fábrica se quiere establecer el tiempo de duración de una nueva bombilla, para ello se escoge una muestra al azar de 30 bombillas y se anota su tiempo de duración en horas, en seguida se muestran los resultados:

\begin{tabular}{|c|c|c|c|c|c|c|c|}
\hline 1000,8 & 830,4 & 12300 & 14962,3 & 11623.2 & 8020,3 & 1592,9 & 9849 \\
\hline 643,9 & 9700 & 11121 & 15000 & 14328,3 & 13002 & 11740,6 & 2535,7 \\
\hline 1287,5 & 14728,6 & 15.200 & 1493,8 & 15000,6 & 12506,4 & 10002,4 & \multirow{1}{*}{} \\
\cline { 1 - 6 } 13621,7 & 2381,6 & 650,9 & 5291,4 & 9983,4 & 9630,9 & 7499,2 & \multicolumn{1}{|c|}{} \\
\cline { 1 - 5 } &
\end{tabular}

Construya la tabla de distribución de frecuencia absoluta que represente la información respecto al tiempo de duración de las bombillas usando intervalos.

6. En una institución educativa se realizó una prueba de matemáticas a 200 estudiantes de grado undécimo con el fin de prepararlos para la aplicación de las Pruebas Saber cuya puntuación máxima es de 100 puntos. Para incentivar a los estudiantes en el desarrollo consiente de la prueba se prometió otorgar el $30 \%$ de descuento de la excursión a los mejores puntajes, mientras que los estudiantes con puntajes más bajos tendrían que asistir a clases de refuerzo y mejoramiento durante todo el año. Teniendo en cuenta que al obtener los resultados de la prueba se realizaron 20 descuentos por el puntaje correspondiente a 90 puntos, que el puntaje más bajo fue de 30 puntos y que fue necesario preparar 50 planes de mejoramiento; y por otro lado que a los 70 estudiantes que obtuvieron 40 puntos se solicitó un certificado de clase particulares para obtener mejores resultados en la prueba; y que los estudiantes restantes obtuvieron la mitad de la puntuación total, para ellos se dispuso un cuestionario que permitiera saber porque solo obtuvieron este puntaje, construya la tabla de frecuencias correspondiente.

\section{APENDICE B. Fragmento de cuestionario para identificación de errores en interpretación de tablas de frecuencias.}

1. A continuación se presenta el tiempo de vida útil de una muestra de 250 televisores producidos por una empresa.

\begin{tabular}{|c|c|c|c|c|}
\hline Vida útil (días) & $\begin{array}{c}\text { Frecuencia } \\
\text { absoluta simple }\end{array}$ & $\begin{array}{c}\text { Frecuencia } \\
\text { relativa simple }\end{array}$ & $\begin{array}{c}\text { Frecuencia absoluta } \\
\text { acumulada }\end{array}$ & $\begin{array}{c}\text { Frecuencia relativa } \\
\text { acumulada }\end{array}$ \\
\hline$[1095-3095)$ & 76 & $76 / 250$ & 76 & $76 / 250$ \\
\hline$[3095-5095)$ & 118 & $118 / 250$ & 194 & $194 / 250$ \\
\hline$[5095-7095]$ & 56 & $56 / 250$ & 250 & $250 / 250$ \\
\hline
\end{tabular}

Con base en la información anterior, responda:

a. ¿Cuál es el tiempo de vida útil de los televisores cuya frecuencia absoluta simple es 118 ?

b. ¿Cuántos televisores tienen entre 3095 y 5095 días de vida útil? 
DOI: $10.20396 /$ zet.v28i0.8656553

c. En el caso de la frecuencia relativa simple ¿Cuántos televisores representan mayor cantidad en cuanto al tiempo en vida útil respecto al total? ¿Por qué?

d. ¿Cuál es el número total de televisores que duran entre 1095 y 5095 días? Explique cómo obtuvo ese valor

2. En un centro comercial se recolectó la siguiente información respecto a las fechas favoritas de 900 personas:

\begin{tabular}{|c|c|c|c|c|}
\hline Fecha & $\begin{array}{c}\text { Frecuencia } \\
\text { absoluta simple }\end{array}$ & $\begin{array}{c}\text { Frecuencia } \\
\text { relativa simple }\end{array}$ & $\begin{array}{c}\text { Frecuencia } \\
\text { absoluta } \\
\text { acumulada }\end{array}$ & $\begin{array}{c}\text { Frecuencia } \\
\text { relativa } \\
\text { acumulada }\end{array}$ \\
\hline Amor y amistad & 178 & $178 / 900$ & 178 & $178 / 900$ \\
\hline Mes del padre & 228 & $228 / 900$ & 406 & $406 / 900$ \\
\hline Mes de la madre & 290 & $290 / 900$ & 696 & $696 / 900$ \\
\hline Halloween & 122 & $122 / 900$ & 818 & $818 / 900$ \\
\hline Navidad & 82 & $82 / 900$ & 900 & $900 / 900$ \\
\hline
\end{tabular}

Responda:

a. Con base en la frecuencia absoluta simple ¿Cuál es la fecha favorita de las personas encuestadas?

b. Con base en la frecuencia relativa simple ¿Cuál es la fecha favorita de las personas encuestadas?

c. Encuentra alguna relación entre las respuestas en los ítems anteriores

d. De acuerdo con la información planteada en la columna de frecuencia relativa simple, en relación con la encuesta ¿Qué época es mejor Halloween o Navidad?

e. ¿Cuántas personas prefieren las fechas especiales anteriores al mes de octubre? ¿Cómo obtuvo el resultado?

f. Con base en las frecuencias relativas ¿las personas que prefieren amor y amistad y mes del padre, representan más respecto al total de las que prefieren Halloween? Explique su respuesta

3. La siguiente tabla presenta información en relación con los ingresos mensuales de 100 familias residentes en la localidad de Engativá. Los resultados se organizaron en cinco categorías, como se muestra en la siguiente tabla.

\begin{tabular}{|c|c|c|c|c|c|}
\hline Categoría & Ingresos (Pesos) & $\begin{array}{c}\text { Frecuencia } \\
\text { absoluta } \\
\text { simple }\end{array}$ & $\begin{array}{c}\text { Frecuencia } \\
\text { relativa } \\
\text { simple }\end{array}$ & $\begin{array}{c}\text { Frecuencia } \\
\text { absoluta } \\
\text { acumulada }\end{array}$ & $\begin{array}{c}\text { Frecuencia } \\
\text { relativa } \\
\text { acumulada }\end{array}$ \\
\hline 1 & {$[700.000-900.000)$} & 12 & $12 / 100$ & 12 & $12 / 100$ \\
\hline 2 & {$[900.000-1100000)$} & 35 & $35 / 100$ & 47 & $47 / 100$ \\
\hline 3 & {$[1.100 .000-1.300 .000)$} & 22 & $22 / 100$ & 69 & $69 / 100$ \\
\hline 4 & {$[1.300 .000-1.500 .000)$} & 18 & $18 / 100$ & 87 & $87 / 100$ \\
\hline 5 & {$[1.500 .000-1.700 .000]$} & 13 & $13 / 100$ & 100 & $100 / 100$ \\
\hline
\end{tabular}

Teniendo en cuenta la información de la tabla anterior, responda:

a. ¿Cuál es el ingreso de las 35 familias que pertenecen a la categoría 2? 
DOI: $10.20396 /$ zet.v28i0.8656553

b. ¿Cuál es el número total de familias que ganan entre $\$ 700000$ y $\$ 1300000$ ? Explique su respuesta.

c. ¿Cuántas familias ganan entre $\$ 1300000$ y $\$ 1500000$ ?

d. Con base en la frecuencia relativa simple, ¿Cuál es el número total de familias que ganan entre $\$ 1500000$ y $\$ 1700000$ ? Explique su respuesta.

e. ¿En qué categoría se encuentran las familias que ganan \$1.100.000? ¿Cuántas familias pertenecen a esta categoría?

4. En la siguiente tabla se muestra información respecto al número de personas reportadas como desaparecidas en los últimos cinco años en Colombia

\begin{tabular}{|c|c|c|c|c|}
\hline Año & $\begin{array}{c}\text { Frecuencia absoluta } \\
\text { simple }\end{array}$ & $\begin{array}{c}\text { Frecuencia relativa } \\
\text { simple }\end{array}$ & $\begin{array}{c}\text { Frecuencia absoluta } \\
\text { acumulada }\end{array}$ & $\begin{array}{c}\text { Frecuencia relativa } \\
\text { acumulada }\end{array}$ \\
\hline 2012 & 5452 & $5452 / 21500$ & 5452 & $5452 / 21500$ \\
\hline 2013 & 3994 & $3994 / 21500$ & 9446 & $9446 / 21500$ \\
\hline 2014 & 3867 & $3867 / 21500$ & 13313 & $13313 / 21500$ \\
\hline 2015 & 4239 & $4239 / 21500$ & 17552 & $17552 / 21500$ \\
\hline 2016 & 3948 & $3948 / 21500$ & 21500 & $21500 / 21500$ \\
\hline
\end{tabular}

Responda:

a. Con base en la tabla, Alison afirma que el 2014 fue el año en el que se reportaron menos desaparecidos en la historia de Colombia ¿Está de acuerdo con ella? ¿Por qué?

b. ¿En qué año, de los incluidos en la tabla, hubo más personas reportadas como desaparecidas?

c. Escriba una conclusión con base en la columna frecuencia absoluta simple de la tabla.

5. En una escuela de patinaje inscribieron a sus estudiantes en un torneo en el cual las categorías se determinan de acuerdo a la edad de los competidores. Los directivos de la escuela reciben el siguiente reporte respecto al rendimiento de sus estudiantes, la valoración se presenta sobre 100 puntos y es el puntaje total obtenido por categoría (no por competidor).

\begin{tabular}{|c|c|c|c|c|}
\hline $\begin{array}{c}\text { Categoría } \\
(\text { edad) }\end{array}$ & $\begin{array}{c}\text { Frecuencia absoluta } \\
\text { simple (Puntos) }\end{array}$ & $\begin{array}{c}\text { Frecuencia } \\
\text { relativa simple }\end{array}$ & $\begin{array}{c}\text { Frecuencia } \\
\text { absoluta acumulada }\end{array}$ & $\begin{array}{c}\text { Frecuencia relativa } \\
\text { acumulada }\end{array}$ \\
\hline$[5-8)$ & 78 & $78 / 320$ & 78 & $78 / 320$ \\
\hline$[8-11)$ & 75 & $75 / 320$ & 153 & $153 / 320$ \\
\hline$[11-14)$ & 86 & $86 / 320$ & 239 & $239 / 320$ \\
\hline$[14-17]$ & 81 & $81 / 320$ & 320 & $320 / 320$ \\
\hline
\end{tabular}

Con base en la información presentada en la tabla anterior responda:

a. Los directivos quieren identificar las edades de los tres competidores que obtuvieron más bajo rendimiento para aumentar su tiempo de entrenamiento, podría decir ¿Quiénes deberán entrenar más tiempo? ¿Por qué?

b. Los directivos quieren identificar la categoría cuyos competidores tuvieron más bajo rendimiento ¿Cuál fue? ¿Por qué?

c. Podría decir cuál fue la categoría en que los competidores tuvieron mejor rendimiento ¿Cuál fue su puntaje? 
d. El próximo torneo se llevará a cabo en la ciudad de Medellín tres meses después de haber recibido los resultados de esta competencia. ¿Podría determinar cuál categoría obtendrá más puntos en dicho torneo? ¿Por qué?

6. Un grupo de adultos mayores (50 años en adelante) ha demandado a la EPS "Salud para todos" alegando que durante la entrega de medicamentos del último mes se dio prioridad a los más jóvenes (personas entre 20 y 49 años). Sin embargo la EPS ha presentado en su defensa la información, respecto a la entrega de medicamentos, según se contempla en la siguiente tabla.

\begin{tabular}{|c|c|c|c|c|}
\hline $\begin{array}{c}\text { Edad } \\
\text { (años) }\end{array}$ & $\begin{array}{c}\text { Frecuencia } \\
\text { absoluta simple }\end{array}$ & $\begin{array}{c}\text { Frecuencia relativa } \\
\text { simple }\end{array}$ & $\begin{array}{c}\text { Frecuencia absoluta } \\
\text { acumulada }\end{array}$ & $\begin{array}{c}\text { Frecuencia relativa } \\
\text { acumulada }\end{array}$ \\
\hline$[20-30)$ & 38 & $38 / 200$ & 38 & $38 / 200$ \\
\hline$[30-40)$ & 42 & $42 / 200$ & 80 & $80 / 200$ \\
\hline$[40-50)$ & 35 & $35 / 200$ & 115 & $115 / 200$ \\
\hline$[50-80]$ & 85 & $85 / 200$ & 200 & $200 / 200$ \\
\hline
\end{tabular}

Con base en la información presentada en la tabla responda

a. ¿Cuántas personas de 30 años reclamaron medicamentos el mes pasado?

b. ¿Cuántas personas entre 20 y 29 años reclamaron medicamentos el mes pasado?

c. ¿Cuántas personas entre 50 y 80 años reclamaron medicamentos el mes pasado?

d. De acuerdo con la cantidad de medicamentos entregados por la EPS, la cual se sustenta en la tabla ¿Respalda usted la postura de los adultos mayores o de la EPS? ¿Por qué?

7. En seguida se presenta una tabla de frecuencias que muestra información respecto a la variable "talla de calzado" de las 190 mujeres adultas que habitan el municipio de Miraflores, Guaviare.

Observe la tabla y responda:

\begin{tabular}{|c|c|c|c|c|}
\hline $\begin{array}{c}\text { Valor de la } \\
\text { variable }\end{array}$ & $\begin{array}{c}\text { Frecuencia } \\
\text { absoluta simple }\end{array}$ & $\begin{array}{c}\text { Frecuencia } \\
\text { relativa simple }\end{array}$ & $\begin{array}{c}\text { Frecuencia absoluta } \\
\text { acumulada }\end{array}$ & $\begin{array}{c}\text { Frecuencia relativa } \\
\text { acumulada }\end{array}$ \\
\hline 34 & 40 & $40 / 190$ & 40 & $40 / 190$ \\
\hline 35 & 37 & $37 / 190$ & 77 & $77 / 190$ \\
\hline 36 & 35 & $35 / 190$ & 112 & $112 / 190$ \\
\hline 37 & 39 & $39 / 190$ & 151 & $151 / 190$ \\
\hline 38 & 39 & $39 / 190$ & 190 & $190 / 190$ \\
\hline
\end{tabular}

a. Suponga que por las fiestas decembrinas el único almacén de calzado de la zona ha agotado todos los zapatos talla 35 para dama. Si sólo 15 de las mujeres cuya talla de calzado es 35 alcanzaron a comprar los zapatos para navidad y todas estaban interesadas en hacerlo. ¿Cuántas se vieron afectadas por la falta de calzado?

b. ¿Cuál es la talla de calzado más alta que debería encargar la tienda de zapatos para preparar sus ventas de fin de año? 